\title{
Application of Three-Dimensional Light Microscopy for Thick Specimen Studies
}

\author{
Yeon Seung Rhyu, Se Jeong Lee ${ }^{1}$, Dong Heui Kim ${ }^{2}$, Chang-Sub Uhm ${ }^{1, *}$ \\ Department of Biotechnology, Korea University College of Life Science \& Biotechnology, Seoul 02841, Korea \\ ${ }^{1}$ Department of Anatomy, Korea University College of Medicine, Seoul 02841, Korea \\ ${ }^{2}$ Department of Environmental Medical Biology, Yonsei University Wonju College of Medicine, Wonju 26426, Korea
}

*Correspondence to:

Uhm CS,

Tel: $+82-2-2286-1150$

Fax: +82-2-929-5696

E-mail: uhmcs@korea.ac.kr

Received May 16, 2016

Revised May 29, 2016

Accepted May 30, 2016
The thickness of specimen is an important factor in microscopic researches. Thicker specimen contains more information, but it is difficult to obtain well focused image with precise details due to optical limit of conventional microscope. Recently, a microscope unit that combines improved illumination system, which allows real time three-dimensional (3D) image and automatic z-stack merging software. In this research, we evaluated the usefulness of this unit in observing thick samples; Golgi stained nervous tissue and ground prepared bone, tooth, and non-transparent small sample; zebra fish teeth. Well focused image in thick samples was obtained by processing z-stack images with Panfocal software. A clear feature of neuronal dendrite branching pattern could be taken. 3D features were clearly observed by oblique illumination. Furthermore, 3D array and shape of zebra fish teeth was clearly distinguished. A novel combination of two channel oblique illumination and $\mathrm{z}$-stack imaging process increased depth of field and optimized contrast, which has a potential to be further applied in the field of neuroscience, hard tissue biology, and analysis of small organic structures such as ear ossicles and zebra fish teeth.

Key Words: Three-dimension, Microscopy, Golgi, Bone, Tooth

\section{INTRODUCTION}

The thickness of specimen is an important factor in microscopic researches. It has been supposed that thicker specimen contains more information, but thinner specimen is of value in providing sharper image. Therefore, section thickness is a critical issue in research design. The soft biological tissues are embedded and cut with microtome according to the goal of study. For routine histological investigation, samples are embedded in paraffin, 2- to 5 - $\mu \mathrm{m}$ thickness sections are cut by microtome, stained, and observed under the light microscope. But, thick sections are still used in biomedical researches to access three-dimensional (3D) information. One example is Golgi stained section, which is used to understand complete morphology of the neuron. The other examples are hard tissue such as bone and tooth. Undecalcified section is appropriate in order to analyze cytoarchitecture of hard tissue. However, it is very difficult to make an optimized section of the undecalcified specimen using microtome. Therefore, these tissues are usually observed after being ground into sections 40 - to 100 - $\mu \mathrm{m}$ thick.

Recently a novel microscopic system has been introduced to get sharp image from thick specimen, which is called "3D Panfocal ${ }^{\mathrm{TM}}$ Microscope" (Edge-3D, USA). This system composed of light microscope, automatic z-axis motorizing system, autofocusing camera system, and computer system for controlling z-stack motor and imaging processing software (Kiely et al., 2011). In this research, we evaluated the usefulness of this unit in observing thick samples such as Golgi stained nervous tissue and ground prepared bone and tooth. As an example of opaque small sample observation, zebra fish teeth were used.

(a) This is an open-access article distributed under the terms of the Creative Commons Attribution Non-Commercial License (http://creativecommons.org/licenses/by-nc/4.0) which permits unrestricted noncommercial use, distribution, and reproduction in any medium, provided the original work is properly cited.

Copyrights @ 2016 by Korean Society of Microscopy 


\section{MATERIALS AND METHODS}

\section{Microscope Unit}

The Edge-3D Research Microscope RM-V3 (Edge-3D) was used in this study. This system is composed of light microscope with transmitted light system and reflected illumination system to facilitate observation of transparent and opaque samples. In addition to main body, it has computer system to control brightness of each light source, automatic $\mathrm{Z}$ motor, camera system, and image processing.

\section{Samples Used in This Study}

\section{Brain Gogli sample preparation}

Mice of c47BL/6 strain were used for this study. The brain was dissected from perfused mice after sodium pentobarbital anesthesia. A rapid Golgi impregnation method of Hama et al. (1989) with slight modifications (Lee et al., 2004) was used. The stained blocks were cut into $100 \mu \mathrm{m}$ thickness sections and re-embedded in the Epon araldite mixture on the glass slides. The sample was under the transmitted light illumination and taken sequential images from top to bottom to construct images stack for deep focus modelling, which is processed by program to construct a single image plane with focus for every stack.

\section{Bone and tooth ground preparation}

The sample slides were selected from slide box prepared for students histology education, Korea University College of Medicine (Seoul, Korea). Selected sample was ground
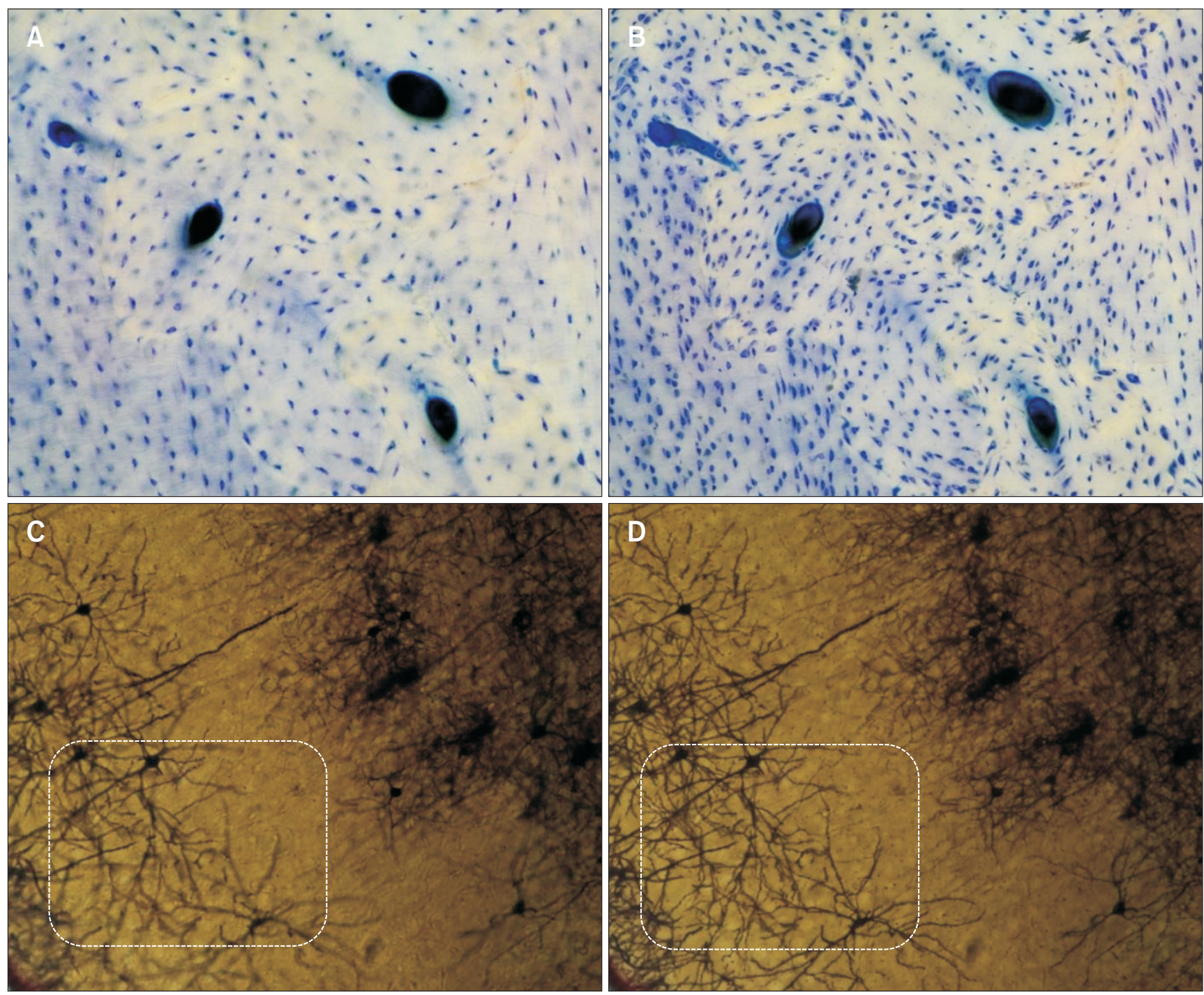

Fig. 1. Effect of z-stack image processing by removal out of focus optical noise. In contrast to single focus image (A, C), panfocal image shows clearly distinguishable details of bone (B) and neuronal dendrites in the indicated area (D). However, image processing may cause distortion of cytoarchitecture because z-stack is compressed into single plane (B). 
prepared human bone and tooth. In order to make bone and tooth's wafer, the sample was sectioned using electro-saw and polished by fine sandpaper. After that, bone was stained with toluidine blue and tooth was attached to the slide without staining. Next, they were observed under transmitted light. In order to produce a fully focused 3D image, multiple stacks of images were acquired by sequential focus optimizing steps from top to bottom section by Edge-3D microscopic system. The image of unstained tooth tissue was also obtained by excluding transmitted light and relying on reflected illumination. Interestingly, interaction between tissue and light showed an informative image which resembles images taken from dark field microscope.

\section{Ankylosed teeth of zebra fish}

Seven-month old zebra fish (Danio rerio) was used in this study. Teeth of zebrafish are located at 5th pharyngeal arch as pair. En bloc dissection of teeth and pharyngeal arch was performed under microsurgery stereoscope and fixed in $4 \%$ paraformaldehyde overnight. After washing the teeth with distilled water, it was stained in $0.5 \%$ alizarin-red solution overnight and washed. The sample was allocated on the slide glass to be observed by optimizing transmission light and reflected illumination while automated z-stack images were collected with $4 \times$ objective lens.

\section{RESULTS}

In this research, newly introduced Edge-3D Research Microscope was applied to observe thick specimens that are often used in biomedical studies. Firstly panfocal imaging system of this unit was applied to study the sample. In Fig. 1A and $\mathrm{B}$, concentrically positioned lamellae with lacunae around the Haversian canal is clearly visible. In contrast to single focus image (Fig. 1A), panfocal image clearly shows features of Haversian canal, lacunae, and osteocytes more distinctly (Fig. 1B). Panfocal image is comprised of focus points of each layers in one plane, so overlapping of lacunae might cause distortion in 3D data (Fig. 1B). Observing image from Golgi stained sample around thickness of $100 \mu \mathrm{m}$, axon and dendritic tree of cortical neuron with partially focused i.e., in and out of focused are observed on the single focus image (Fig. 1C). However, using panfocal imaging technique, axon and dendrite of neuron cell are clearly distinguished (Fig. 1D).

Golgi stained section for neurohistology research was observed by $40 \times$ object lens under a single focus, but in these cases some dendrites are out of focus (Fig. 2A). However, using panfocal imaging module, it was able to clearly identify dendrite tree branching out from a neuron (Fig. 2B). One prominent feature of this microscope unit is merging left and right image into stereo paired image that shows dendrite tree branching out in 3D directions. With little practice, 3D perception is possible after merging left (Fig. 2C) and right (Fig. 2D) image in the brain. Alternatively anaglyph image (Fig. 2E) make us recognize 3D information by using blue/red glasses used for 3D television.

By observing cortical bone using panfocal imaging system, Harversian system and lamella patterns are more clearly demonstrated compared to single focus imaging (Fig. 3A and B). Also, bone image taken by stereo imaging system

\section{A. Single focus image \\ B. Panfocal image \\ C. Left stereo image \\ D. Right stereo image \\ E. Anaglyphic image}

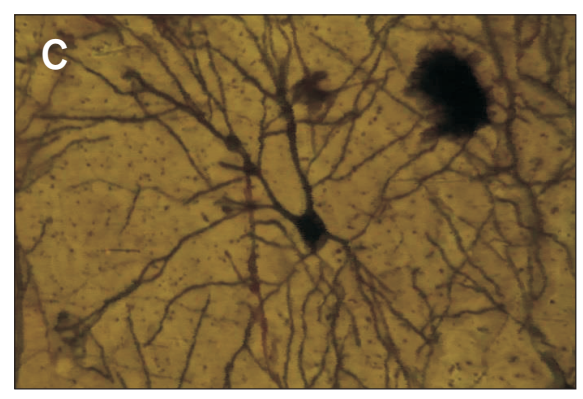

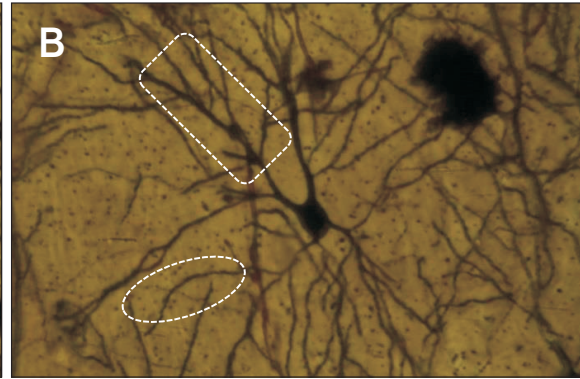
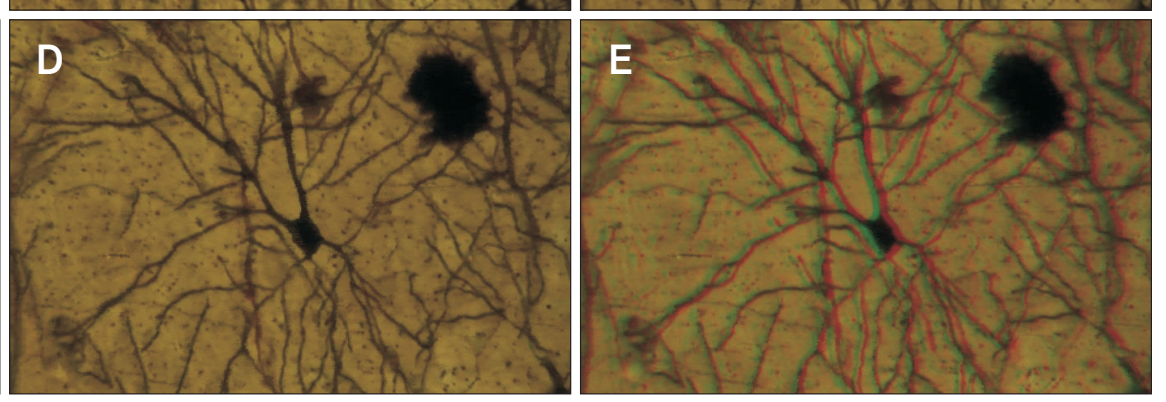

Fig. 2. Neuronal image taken with $40 \times$ objective lens. Neuronal morphology is distinctively seen at (B) while three-dimensional of dendrite is clearly observed in stereo pair image (C, D). The panfocal processing effect is obvious in rectangular and oval zone of Fig. 2A and B. i.e., the blurred dendrite on the Fig. 2A become sharp on the Fig. 2B. Also, stereoscopic features of dendritic trees can be observed under red-blue glasses (E). 
A. Single focus image

B. Panfocal image

C. Left stereo image

D. Right stereo image

E. Anaglyphic image
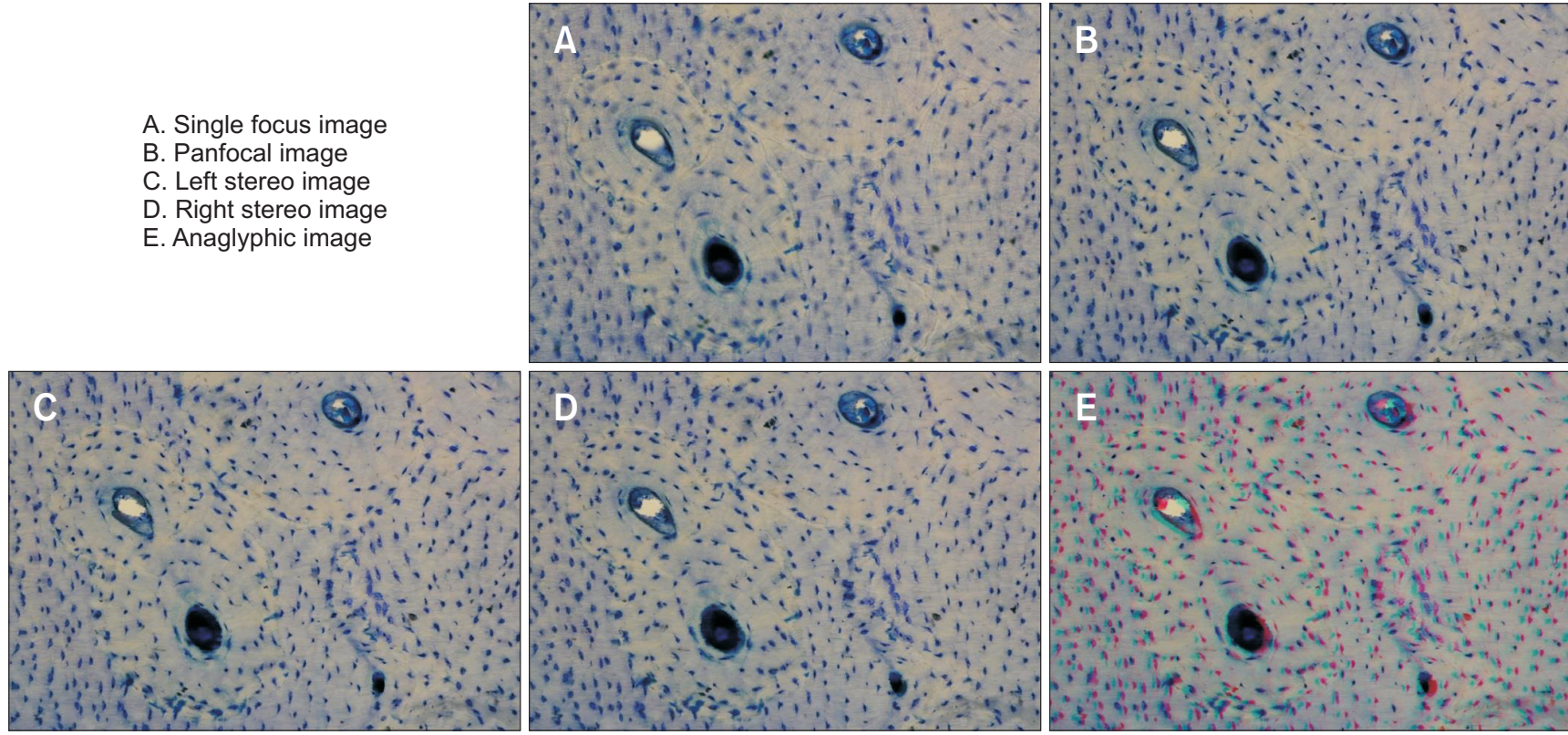

Fig. 3. Organization of Harversian system in the compact bone observed with $20 \times$ objective lens (A, B). Three Harversian systems are observed. Well focused concentric lacunae can be clearly identified aligned around the Harversian canal (B). Three-dimensional organization of the osteon are better demonstrated under the stereo-paired view (C, D) and red-blue glasses (E).

A. Single focus image

B. Panfocal image

C. Left stereo image

D. Right stereo image

E. Anaglyphic image
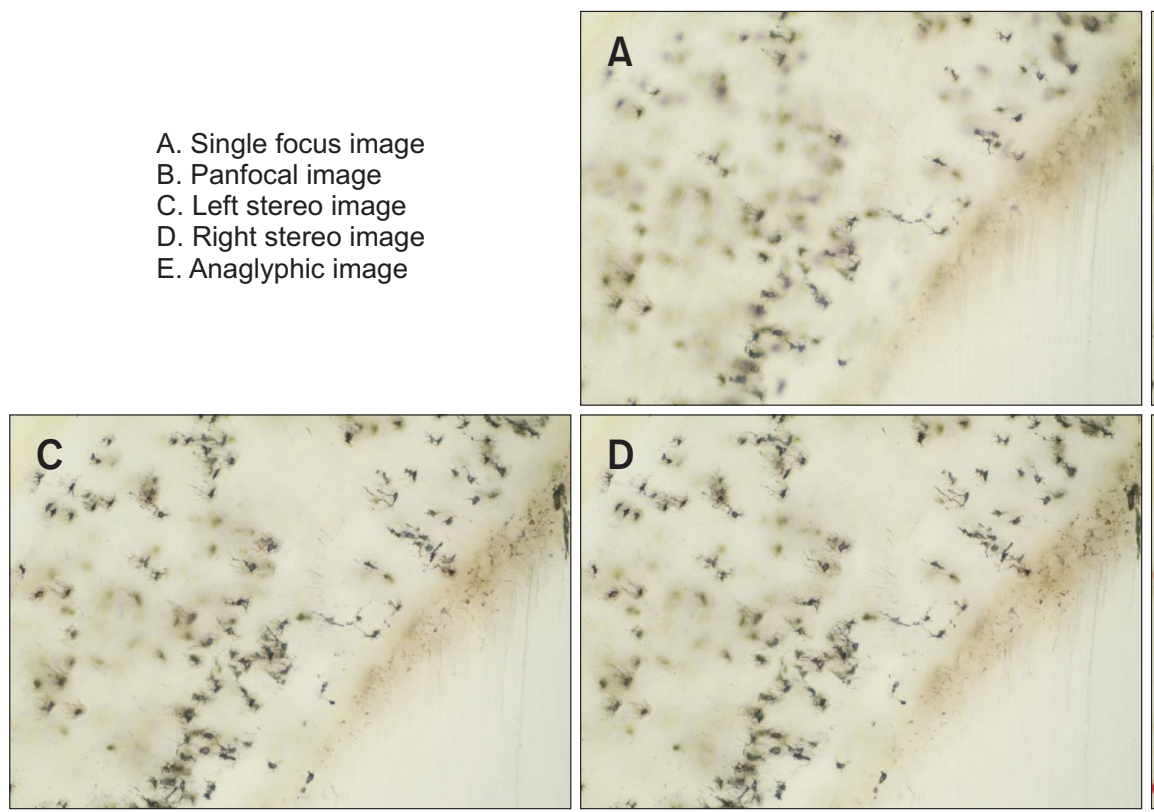
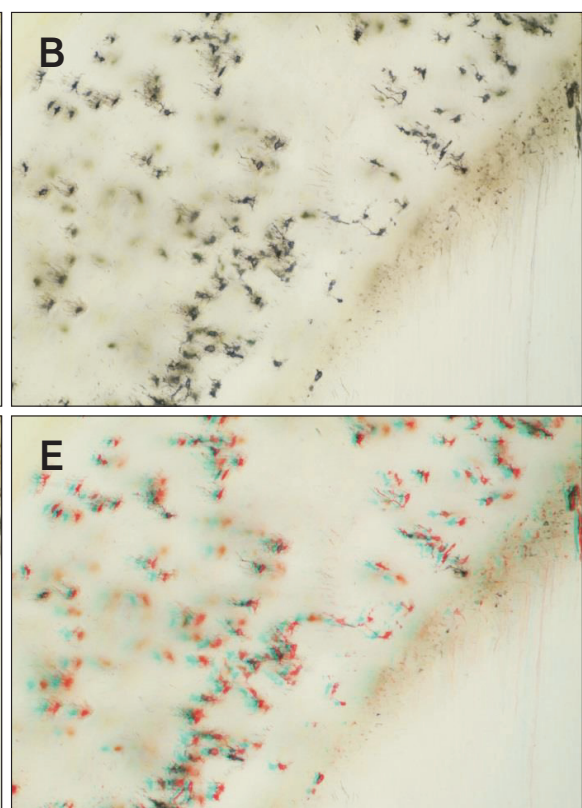

Fig. 4. Image at the junction between dentin and cementum of the tooth root taken with $20 \times$ objective lens under transmitted illumination. Improved image (B) is observed after panfocal image processing, which shows more clear image compared to single focused image (A). Three-dimensional formation of the dentinal tubules, glomerulus layer, and lacunae near cementocytes are well demonstrated under the stereo-paired view (C, D) and red-blue glasses (E).

shows osteocytes within lacunae around Harversian canal. Furthermore, diverse directional orientation of osteocyte cells are observed (Fig. 3C-E). Even more, overlapping distortion as a result of panfocal process can be corrected (Fig. 1B) by observing stereo imaging investigation (Fig. 3C-E).
Alike bone, tooth is one of the hardest tissue in body. Therefore, ground preparation technique is the most typical method to study cytoarchitecture of the tooth. Fig. 4 is an image obtained at the boundary between dentin and cementum. In a single focus image, lacunae of cementocytes 
and canaliculi are observed, while tubule can be seen on dentin layer (Fig. 4A). Panfocal image (Fig. 4B) exhibits more lacunae of cementocytes, and canaliculi are observed more clearly compared to former image (Fig. 4A). By applying stereo imaging system, 3D arrangement of lacuna of cementocyte and its canaliculi can be observed. Also,
Tomes' granular layer is clearly distinguished, where dentinal tubule ends (Fig. 4C-E). Conventional light microscope uses transmitted light to gain image of the sample while Edge3D Research Microscope has additional reflected light from both sides of stage. Fig. 5 shows interesting image provided by interaction between reflected light and tooth tissue (Fig.
A. Single focus image

B. Panfocal image

C. Left stereo image

D. Right stereo image

E. Anaglyphic image

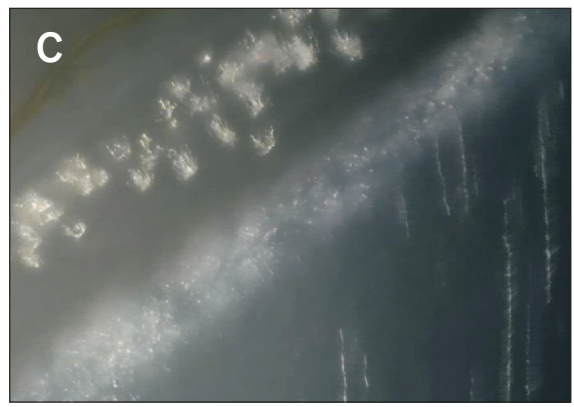

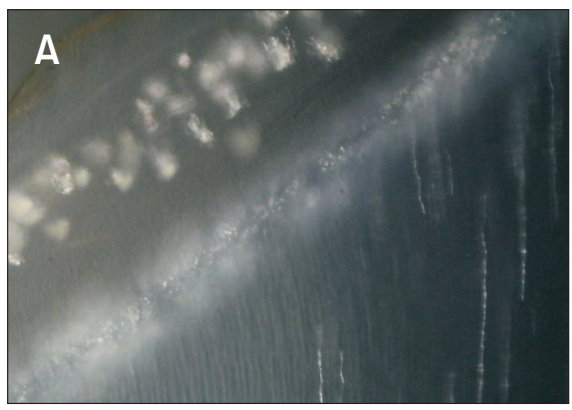
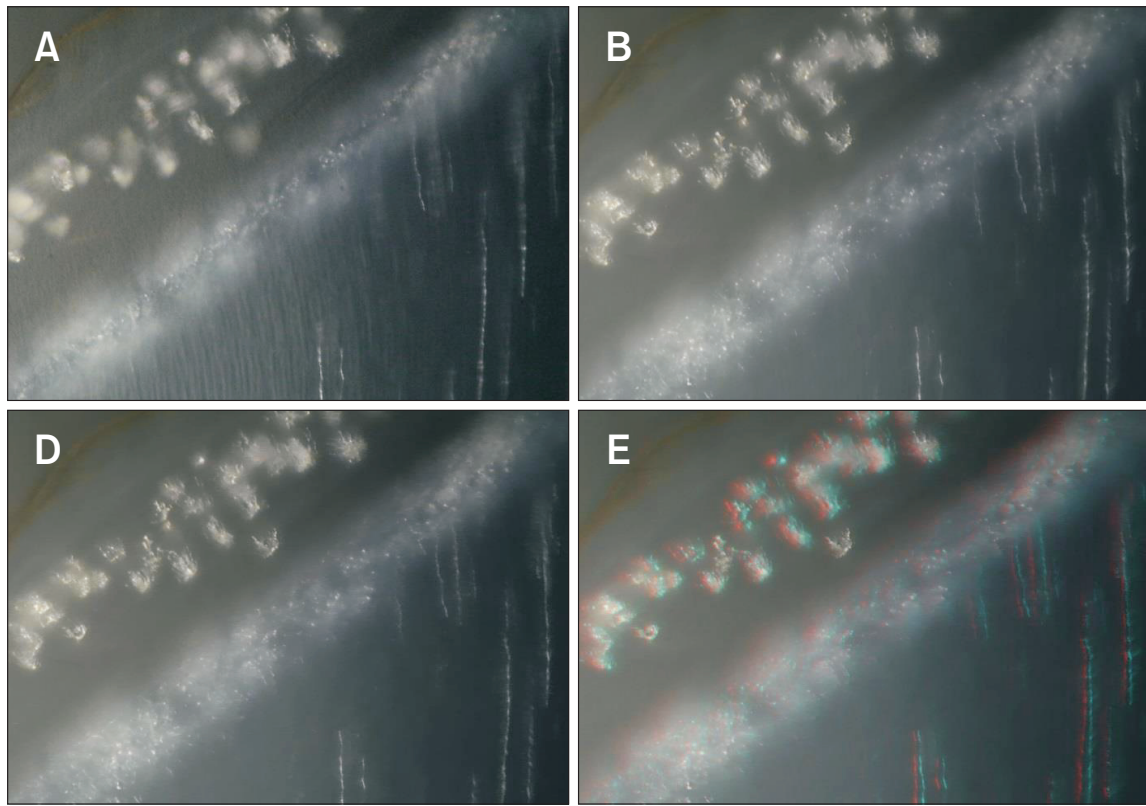

Fig. 5. (A-E) Image at the junction between dentin and cementum of the tooth root taken with $20 \times$ objective lens under reflected illumination. The reflected light without transmitted light revealed unique images similar to dark field microscopy. The dentinal tubules and glomerulus layer in the dentin are clearly observed. The lacunae for cementocytes and their canaliculi are well demonstrated under the three-dimensional microscope.

A. Single focus image

B. Panfocal image

C. Left stereo image

D. Right stereo image

E. Anaglyphic image
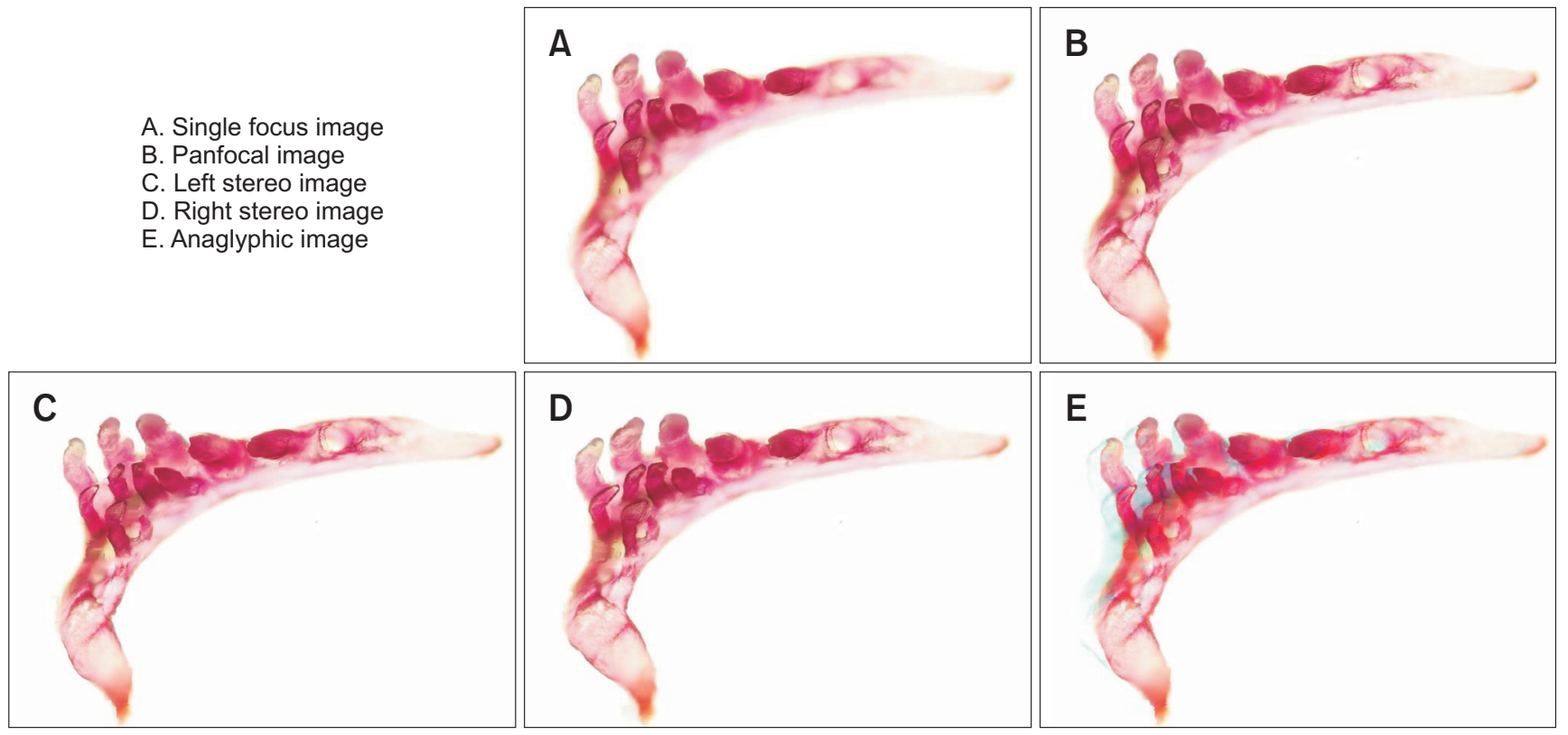

Fig. 6. Teeth ankylosed in the 5 th pharyngeal arch of the zebrafish taken with $4 \times$ objective lens (A). The three-dimensional microscopic system effectively captures 11 teeth in three rows from ventral to dorsal. The result of panfocal processing provides focus for each teeth in one image, allowing appropriate image for analysis of tooth structure (B). (C-E) Three-dimensional formation of teeth are well distinguishable under the three-dimensional microscope. 
5). Especially, detailed structure of lacunae, canaliculi and dentinal tubule are clearly distinguished (Fig. 5C-E). Observation of non-transparent sample using Edge-3D Research Microscope has been introduced in this study. Zebrafish tooth is widely used in the studies of developmental biology. In this study, zebrafish tooth was three-dimensionally observed by Edge-3D Research Microscope, indicating possibility for further application of the device. Additionally, images were taken by optimizing transmitted light and reflected light, identifying the best contrast for image processing. It is possible to observe zebrafish teeth by single focus image (Fig. 6A), but focus for each teeth differs. On the other hand, by applying panfocal imaging method, each 3 rows of teeth area are all focused and clearly identified (Fig. $6 \mathrm{~B}$ ). We can clearly see three rows of teeth three-dimensionally and their detailed morphology (Fig. 6C-E).

\section{DISCUSSION}

Thick specimens have been used in biological researches to understand 3D configuration of cytoarchitecture and to overcome technical limitation in making sufficient thin sections appropriate for routine microscopic observation. Typically, in order to examine thick sample, assuming overall structure by modifying focus for each point or using system such as camera lucida is used. The panfocal imaging processing eliminates out of optical noise from being out of focus by specialized algorithms that clarify very thick sections and samples. The integrated final result resemble voxel based datarich 3D image obtained by confocal microscope. Pan focal image does assist analyzing thick sample by forming well focused image, but causes distortion of overall cell formation when z-stacks are co-existent (Fig. 1B). Therefore, additional observation of 3D stereo-image is recommendable.

One strategy to effectively observe thick specimen is to increase the depth of field. Oblique illumination increases depth of field without reducing resolution. The depth of field can be doubled by using oblique illumination (Greenberg \& Boyde, 1997). At the same time, oblique illumination increase contrast by producing highlights and shadows in the specimen. The oblique illumination system is formed by placing pyramid mirror at illumination system. Also, real time 3D observation is possible by installing two channel illumination system that controls two light source in a way that one channel goes to left eye and the other going to right eye. Practically, these two lights rapidly alternate and each image is presented to the left and right eyes accordingly, using the $3 \mathrm{D}$ glasses. 3D image obtained by this method will provide sufficient depth of field for precise image analysis. Using these characteristics of 3D microscopy, Golgi stained block was observed together with samples such as neuron, glia, and blood vessel (Greenberg \& Boyde, 1993). A research has introduced a method that increases accuracy of pap smear pathology by applying 3D microscopy (Ramsamooj et al., 2002). In this research, well focused image was obtained from not only neuron tissue but also from hard tissues in body such as bone and teeth, so further application to hard tissue research seems to be possible.

Interesting feature of Edge-3D Research Microscope is that it is possible to turn on and off transmitted light and reflected light according to condition while brightness of each light can be adjusted to gain optimized contrast for characteristics of the specimen. In this research, an interesting image of tooth was obtained by tuning transmitted light off and using low angle of reflected light, which means this method has possibility to be applied on further studies of hard tissue such as tooth or bone. Also, there are former studies that observed lunar regolith, sand, concrete crack (Kiely et al., 2011; Nemati et al., 1998). According to the observations above, we assumed that zebra fish teeth structure, which is a biological sample, was possible to observe. Teeth of mature zebra fish are complex structure of $500 \mu \mathrm{m}$ that consists 11 teeth (Wiweger et al., 2012). Recently zebra fish is used as an animal model in various studies such as development of tooth and research for effect of ecologic pollutants (Bartlett et al., 2005; Wiweger et al., 2012). In these studies high resolution stereoscope and scanning electron microscope were used to examine effects of variances on tooth. In this research, $4 \times$ objective lens was used for study, but if $10 \times$ objective lens is to be used, it is possible to be an effective method to precisely analyze each tooth.

\section{CONCLUSIONS}

The depth of field is a critical issue in microscopic research, especially when the specimen is thick. By combining panfocal imaging processing algorithms, real time 3D light microscopic module, and two illumination systems of transmitted and reflected light, it is possible to obtain accurate image of thick specimen. Therefore, this system would be a useful tool to investigate thick specimens in biomedical researches.

\section{CONFLICT OF INTEREST}

No potential conflict of interest relevant to this article was reported. 


\section{REFERENCES}

Bartlett J D, Dwyer S E, Beniash E, Skobe Z, and Payne-Ferreira T L (2005) Fluorosis: a new model and new insights. J. Dent. Res. 84, 832-836.

Greenberg G and Boyde A (1993) Novel method for stereo imaging in light microscopy at high magnifications. Neuroimage 1, 121-128.

Greenberg G L and Boyde A (1997) Convenient and controllable directview 3D imaging in conventional light microscopes: approaches via illumination and inspection. Proc. Roy. Microsc. Soc. 32, 87-100.

Hama K, Arii T, and Kosaka T (1989) Three-dimensional morphometrical study of dendritic spines of the granule cell in the rat dentate gyrus with HVEM stereo images. J. Electron. Microsc. Tech. 12, 80-87.

Kiely C, Greenberg G, and Kiely C J (2011) A new look at lunar soi collected from the sea of tranquility during the Apollo 11 mission. Microsc. Microanal. 17, 34-48.

Lee K J, Kim H, Kim T S, Park S H, and Rhyu I J (2004) Morphological analysis of spine shapes of Purkinje cell dendrites in the rat cerebellum using high-voltage electron microscopy. Neurosci. Lett. 359, 21-24.

Nemati K M, Monteiro J M, and Scrivener K L (1998) Analysis of compressive stress-induced cracks in concrete. ACI Mater. J. 95, 617-630.

Ramsamooj R, Doolin E, Greenberg G, Catalano E, and Hewitt C W (2002) Real-time, high-definition, three-dimensional microscopy for evaluating problematic cervical Papanicolaou smears classified as atypical squamous cells of undetermined significance. Cancer $\mathbf{9 6}$, 181-186.

Wiweger M I, Zhao Z, van Merkesteyn R J, Roehl H H, and Hogendoorn P C (2012) HSPG-deficient zebrafish uncovers dental aspect of multiple osteochondromas. PLoS One 7, e29734. 